\title{
Comparative outcome assessment of epidermal growth factor receptor tyrosine kinase inhibitors for the treatment of advanced non-small-cell lung cancer: a network meta-analysis
}

\author{
Ramon Andrade De Mello ${ }^{1,2,3}$, Carles Escriu ${ }^{4,5}$, Pedro Castelo-Branco ${ }^{1,2,6}$, Paloma \\ Lucena Cabral7, Giannis Mountzios ${ }^{8}$, Gilberto de Lima Lopes ${ }^{9}$ and Pedro Madureira ${ }^{10}$ \\ ${ }^{1}$ Division of Oncology, School of Medicine, Department of Biomedical Sciences and Medicine, University of Algarve, Faro, \\ Portugal \\ ${ }^{2}$ Algarve Biomedical Center, Campus Gambelas, Faro, Portugal \\ ${ }^{3}$ Research Centre/Department of Medical Oncology, Haroldo Juaçaba Hospital, Ceará Cancer Institute, Fortaleza, CE, Brazil \\ ${ }^{4}$ Department of Medical Oncology, The Clatterbridge Cancer Centre NHS Foundation Trust, Warrington, Wirral and Liverpool, \\ Merseyside, United Kingdom \\ ${ }^{5}$ Cancer Research Centre, Department of Molecular and Clinical Cancer Medicine, The University of Liverpool, Liverpool, \\ United Kingdom \\ ${ }^{6}$ Centre for Biomedical Research, University of Algarve, Faro, Portugal \\ ${ }^{7}$ Special Training Program (PET), Faculty of Medicine, Federal University of Ceará, Fortaleza, CE, Brazil \\ ${ }^{8}$ Department of Medical Oncology, University of Athens, Athens, Greece \\ ${ }^{9}$ Sylvester Comprehensive Cancer Centre at the University of Miami, Miami, FL, USA \\ ${ }^{10}$ Institute for Molecular and Cell Biology (IBMC) and Institute for Investigation and Innovation in Health (i3S), University \\ of Porto, Porto, Portugal
}

Correspondence to: Ramon Andrade De Mello, email: ramondemello@gmail.com

Keywords: non-small cell lung cancer; epidermal growth factor receptor; tyrosine kinase inhibitors

Received: June 20, 2017 Accepted: October 30, $2017 \quad$ Published: December 23, 2017

Copyright: De Mello et al. This is an open-access article distributed under the terms of the Creative Commons Attribution License 3.0 (CC BY 3.0), which permits unrestricted use, distribution, and reproduction in any medium, provided the original author and source are credited.

\section{ABSTRACT}

Introduction: Tyrosine kinase inhibition of the epidermal growth factor receptor (EGFR) is the standard in the first line treatment of patients with advanced nonsmall-cell lung cancer (NSCLC) harbouring EGFR activating mutations. Here we aim to discern efficacy and toxicity measures through a meta-analysis of published studies that could aid treatment selection.

Materials And Methods: We performed a meta-analysis of the main randomized clinical trials evaluating the currently approved EGFR-TKIs in first-line of treatment of EGFR-positive advanced NSCLC. Cochrane guidelines were used for statistical analysis.

Results: 3,179 patients were included. All EGFR TKIs showed improved outcomes with respect to ORR and PFS when compared to standard platinum-doublet

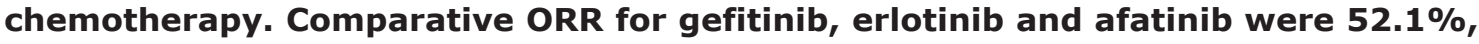
$67.3 \%$ and $61.6 \%$ respectively. HRs for PFS were $0.62(95 \% \mathrm{CI}, 0.38-1.00)$ for gefitinib, 0.28 ( $95 \% \mathrm{CI}, 0.17-0.45)$ for erlotinib and 0.40 (95\% CI, $0.20-0.83$ ) for afatinib. HRs for OS were not statistically significant for any agent.

Conclusions: Our results suggest similar clinical efficacy and higher toxicity of Afatinib treatment. As this still remains the agent with best CSF penetration, we suggest its use is limited to patients presenting with brain metastasis. We suggest the use of Gefitinib in patients without CNS involvement. Faced with the impossibility to dose-reduce Gefitinib, Erlotinib represents a tolerable and effective alternative to Afatinib and Gefitinib if response to EGFR inhibition is considered still effective. 


\section{INTRODUCTION}

Non-small-cell lung cancer (NSCLC) is the major cause of cancer-related death worldwide [1]. The Epidermal Growth Factor Receptor (EGFR), a transmembrane glycoprotein, is mutated in approximately $10-15 \%$ of European patients, more frequently in women, adenocarcinoma type and never-smokers [2]. When the EGFR gene is mutated, (most commonly with exon 19 deletions or exon 21 L858R point mutation), constitutive receptor activation influences the cell cycle, the apoptotic pathway and the production of inflammatory agents [3]. This understanding of EGFR signalling led to the development of specific tyrosine-kinase inhibitors (TKIs) [4], which reached three generations: gefitinib and erlotinib (first); afatinib, dacomitinib, and neratinib (second); rociletinib, HM61713, osimertinib and others (third). The last generation overcomes the threonineto-methionine substitution (T790M) in exon 20 of the EGFR gene, responsible for $50 \%$ of resistance mechanisms to first line anti-EGFR therapy with first and second generation agents [5]. Only gefitinib, erlotinib, and afatinib are approved by Food and Drug Administration (FDA) thus far for the first line setting [6-8].

In patients whose tumours harbours an activating EGFR mutation, EGFR TKIs should be used as firstline therapy [6-9], whereas for the rest of NSCLC cases, standard treatment currently consists of platinum-based doublet chemotherapy. Gefitinib, erlotinib and afatinib show higher response rates and longer progression free survival than chemotherapy in those patients, as tested in several clinical trials exhibiting consistent results [10-20], all of them favouring the target therapy.

Since there are several similar drugs targeting the EGFR mutation in NSCLC first line setting, the critical question emerging is which one should be best for this setting. Our analysis presents the findings of a network meta-analysis, attempting to access the main outcomes among EGFR TKIs in NSCLC, exploiting the data of clinical trials with gefitinib, erlotinib and afatinib. Recently, the Lux-Lung 7 study reported longer PFS and similar OS when comparing Afatinib with Gefitinib, but a triple arm comparison of all these agents is unlikely to occur. Here we aimed to provide an indirect comparison among these drugs which may contribute to guide the drug choice for physicians.

\section{MATERIALS AND METHODS}

For this comparative meta-analysis, we performed computerized searches of the Medline. Embase, Scopus and Information Sciences Institute (ISI) databases up to August 14, 2016, using the following terms: "gefitinib" OR "afatinib" OR "erlotinib" AND "NSCLC" OR "lung cancer" OR "epidermal growth factor". These searches were complemented by examining review articles. Only articles published in English, available in full text and reporting results of randomized, double-arm, phase III clinical trials comparing EGFR-TKIs with chemotherapy regimens were included. The most recent -updateddata of the studies were used for the meta-analysis. For gefitinib, erlotinib and afatinib, only first line treatments were considered due to the paucity of trials comparing these agents to chemotherapy in second line. There were no time restrictions in the search. Exclusion criteria were: trials with patients presenting Eastern Cooperative Oncology Group (ECOG) performance status $>2$ and those including EGFR TKI plus chemotherapy versus chemotherapy (Effectiveness of EGFR-TKIs may be obscured in this setting). Case reports or patient series, which report few patients, were excluded. All abstracts were screened twice and unrelated studies were excluded.

For included trials, we extracted data on: title, first author, year of publication, study design (inclusion and exclusion criteria), patient's characteristics (median patient age, stage of disease, performance status, gender, smoking status, histology, tissue-assessed EGFR mutation), treatment schedules and line of treatment, outcomes from the trial, incidence of adverse events, demographic data. If the study was updated, main outcomes were extracted from the last published article. Data extraction was done independently by two of the authors and divergences were resolved by consensus with a third author.

The primary outcome of this meta-analysis was objective response rate (ORR). Second outcomes were progression free survival (PFS), overall survival (OS) and incidence of adverse events (AE). Summary measures were risk ratio ( $95 \%$ confidence interval [CI]; 95\% PI) for ORR and AE and hazard ratio for OS and PFS.

ORR was defined as the proportion of patients who presented complete or partial response, assessed by Response Evaluation Criteria in Solid Tumours (RECIST) [21] in most of the studies. The time of assessment varied for each trial. PFS was the time, in months, from the randomization until disease progression, or death. OS was the time, in months, from the randomization to death. $\mathrm{AE}$ could be any unfavourable and unintentional sign, symptom, or disease temporarily associated with the use of the drugs, without any judgment about causality or relationship to them. Relevant adverse events of all grades related by two or more studies were condensed by each EGFR TKI arm and compared as meta-estimation with another EGFR TKI.

Statistical analysis was directed by Cochrane Guidelines [22]. We combined the risk ratios from each study using the random-effects model (Mantel-Haenzsel) [23]. For the hazard ratios, the Inverse Variance method was used. The heterogeneity between trials was estimated by the $I^{2}$ statistic. We used the Review Manager version 5.3.5. 


\section{RESULTS}

As shown in the flow chart of the meta-analysis (Figure 1), 09 eligible studies were identified. All of them were included in the current analysis (Table 1), totalizing 3,179 patients. NEJ002 [15]; WJTOG3405 [16], FirstSIGNAL [17], and IPASS [14] evaluated gefitinib as first-line treatment to, respectively, carboplatin plus paclitaxel, cisplatin plus docetaxel, cisplatin plus gemcitabine, and carboplatin plus paclitaxel; LUXLung 3 [24] and LUX-Lung 6 [19] compared afatinib as first-line treatment with cisplatin plus pemetrexed and gemcitabine, respectively. EURTAC [20], OPTIMAL [12] and ENSURE [25] compared first-line erlotinib with cisplatin plus docetaxel, gemcitabine plus carboplatin, and cisplatin plus gemcitabine, respectively. NEJ002, IPASS, and OPTIMAL published updated outcomes, so 12 reports were used in total for this meta-analysis.
Patients' characteristics are summarized in Table 2. More patients were female $(2,315$ of 3,179 $[72.8 \%])$, never smokers $(2,606$ of $3,179[81.9 \%])$, with performance status from 0 to 1 (2,974 of $3,179[93.5 \%])$ and had tumours of adenocarcinoma histology (3,068 of 3,179 [96.5\%]). Disease stage was not summarized because of differences in evaluation among studies.

The risk ratio of objective response rate (ORR) is shown in Figure 2. For gefitinib versus chemotherapy as first-line treatment, $52.1 \%$ (476 out of 913) of patients treated with gefitinib showed complete or partial response against $34.2 \%$ (311 out of 910) of patients treated with chemotherapy, and the pooled risk ratio (RR) was 1.69 (95\% CI, 1.31-2.19; $p<0.0001)$. For afatinib versus chemotherapy, RR was $2.70(95 \% \mathrm{CI}$, $2.12-3.45 ; p<0.0001) ; 61.6 \%$ (291 of 472 ) of patients in the afatinib arm had response, compared to $22.8 \%$

\section{Records identified through database research $(n=4061)$}

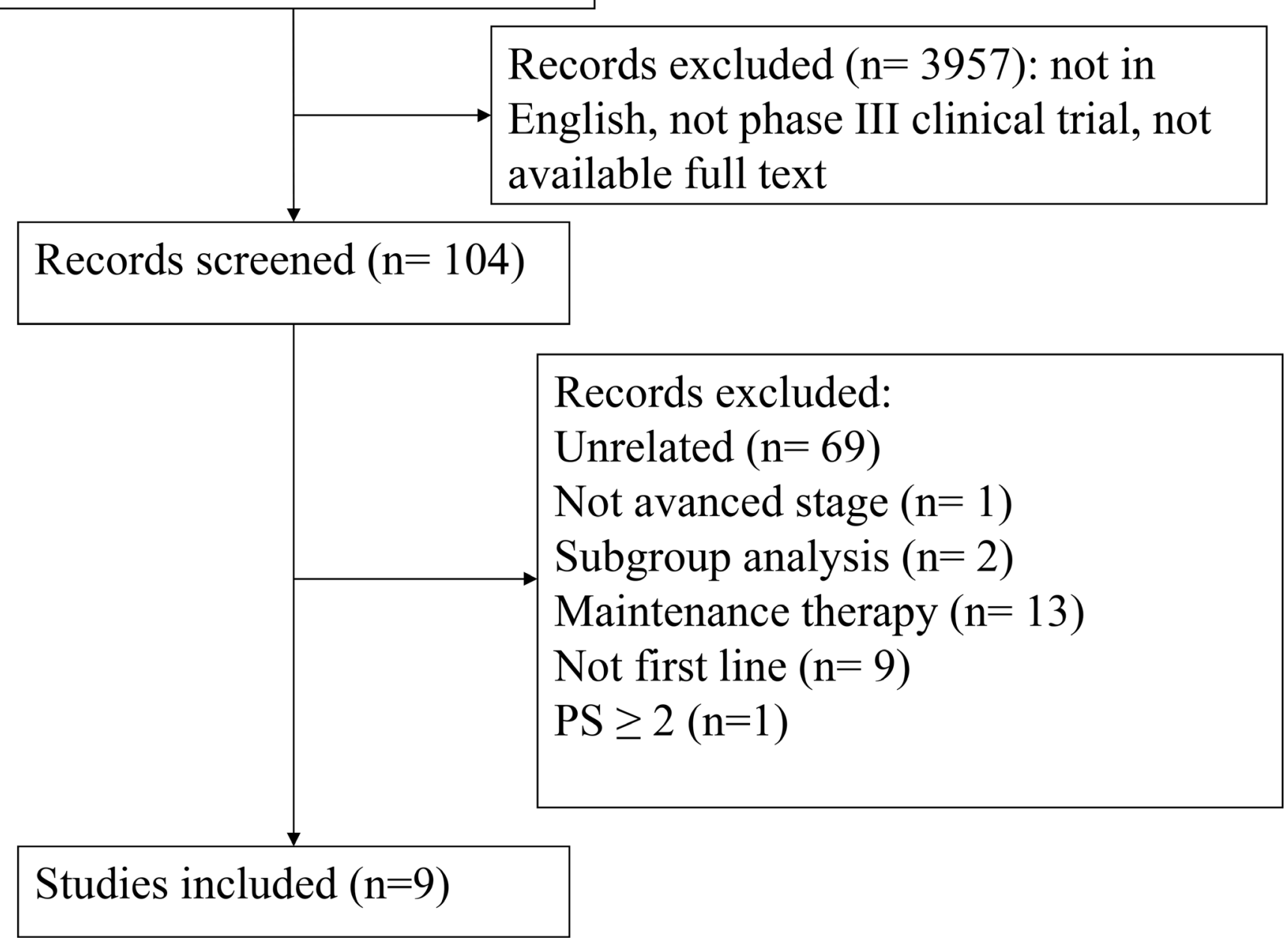

Figure 1: Study selection. 
Table 1: Patient demographics and disease characteristics of included studies

\begin{tabular}{|c|c|c|c|c|c|}
\hline Study & $\begin{array}{l}\text { First } \\
\text { author }\end{array}$ & Population & Line & Treatment arms & $\begin{array}{l}\text { Response } \\
\text { criteria }\end{array}$ \\
\hline $\begin{array}{l}\text { ENSURE } \\
(2015)\end{array}$ & $\mathrm{Wu}$ & $\begin{array}{l}\text { Chemotherapy-naïve patients from China, Malaysia, and the } \\
\text { Philippines with stage } \\
\text { IIIB/IV EGFR mutation-positive NSCLC }\end{array}$ & First & $\begin{array}{l}\text { Erlotinib } 150 \mathrm{mg} / \text { day }(n=110) \\
\text { Gemcitabine } 1000 \mathrm{mg} / \mathrm{m}^{2} \text { plus cisplatin } 75 \mathrm{mg} / \mathrm{m}^{2} \\
\text { every } 3 \text { weeks }(n=107)\end{array}$ & RECIST \\
\hline $\begin{array}{l}\text { LUX-Lung } 6 \\
(2014)\end{array}$ & $\mathrm{Wu}$ & $\begin{array}{l}\text { Patients with previously untreated stage IIIB or IV lung } \\
\text { adenocarcinoma and EGFR mutation-positives }\end{array}$ & First & $\begin{array}{l}\text { Afatinib } 40 \mathrm{mg} / \text { day }(n=242) \\
\text { Gemcitabine } 1000 \mathrm{mg} / \mathrm{m}^{2} \text { plus cisplatin } 75 \mathrm{mg} / \mathrm{m}^{2} \\
\text { every } 3 \text { weeks }(n=122)\end{array}$ & RECIST \\
\hline $\begin{array}{l}\text { LUX-Lung } 3 \\
(2013)\end{array}$ & Sequist & $\begin{array}{l}\text { Treatment-naïve patients with advanced lung adenocarcinoma } \\
\text { and EGFR mutation-positives }\end{array}$ & First & $\begin{array}{l}\text { Afatinib } 40 \mathrm{mg} / \text { day }(n=230) \\
\text { Cisplatin } 75 \mathrm{mg} / \mathrm{m}^{2} \text { and pemetrexed } 500 \mathrm{mg} / \mathrm{m}^{2} \\
(n=115)\end{array}$ & RECIST \\
\hline $\begin{array}{l}\text { EURTAC } \\
(2012)\end{array}$ & Rosell & $\begin{array}{l}\text { European patients with stage IIIB or IV NSCLC and EGFR } \\
\text { mutations who had no history of chemotherapy for metastatic } \\
\text { disease }\end{array}$ & First & $\begin{array}{l}\text { Erlotinib } 150 \mathrm{mg} / \text { day }(n=86) \\
\text { Cisplatin } 75 \mathrm{mg} / \mathrm{m}^{2} \text { plus docetaxel } 75 \mathrm{mg} / \mathrm{m}^{2} \text { or } \\
\text { cisplatin } 75 \mathrm{mg} / \mathrm{m}^{2} \text { plus gemcitabine } 1250 \mathrm{mg} / \mathrm{m}^{2} \\
(n=87)\end{array}$ & RECIST \\
\hline $\begin{array}{l}\text { First- } \\
\text { SIGNAL } \\
(2012)\end{array}$ & Han & $\begin{array}{l}\text { Chemotherapy-naïve and never-smokers patients with stage IIIB } \\
\text { or IV adenocarcinoma of the lung }\end{array}$ & First & $\begin{array}{l}\text { Gefitinib } 250 \mathrm{mg} / \text { day }(n=159) \\
\text { Gemcitabine } 1,250 \mathrm{mg} / \mathrm{m}^{2} \text { plus cisplatin } 75 \mathrm{mg} / \mathrm{m}^{2} \\
\text { every } 3 \text { weeks }(n=150)\end{array}$ & WHO \\
\hline $\begin{array}{l}\text { OPTIMAL } \\
(2011)\end{array}$ & $\begin{array}{l}\text { Zhou and } \\
\mathrm{Wu}\end{array}$ & $\begin{array}{l}\text { Chinese patients with stage IIIB or IV NSCLC and a confirmed } \\
\text { activating mutation of EGFR, without receiving therapy for } \\
\text { metastatic disease }\end{array}$ & First & $\begin{array}{l}\text { Erlotinib } 150 \mathrm{mg} / \text { day }(n=82) \\
\text { Gemcitabine } 1000 \mathrm{mg} / \mathrm{m}^{2} \text { plus carboplatin AUC }=5 \\
\text { every } 3 \text { weeks }(n=72)\end{array}$ & RECIST \\
\hline $\begin{array}{l}\text { NEJ002 } \\
(2010)\end{array}$ & Maemondo & $\begin{array}{l}\text { Japanese patients with metastatic NSCLC } \\
\text { and EGFR mutations who had not previously received } \\
\text { chemotherapy }\end{array}$ & First & $\begin{array}{l}\text { Gefitinib } 250 \mathrm{mg} / \text { day }(n=114) \\
\text { Carboplatin AUC }=6 \text { plus paclitaxel } 200 \mathrm{mg} / \mathrm{m}^{2} \\
\text { every } 3 \text { weeks }(n=114)\end{array}$ & RECIST \\
\hline $\begin{array}{l}\text { WJTOG } 3405 \\
(2009)\end{array}$ & Mitsudomi & $\begin{array}{l}\text { Patients with advanced or recurrent NSCLC harbouring an } \\
\text { activating mutation of the EGFR }\end{array}$ & First & $\begin{array}{l}\text { Gefitinib } 250 \mathrm{mg} / \text { day }(n=88) \\
\text { Cisplatin } 80 \mathrm{mg} / \mathrm{m}^{2} \text { plus docetaxel } 60 \mathrm{mg} / \mathrm{m}^{2}(n=89)\end{array}$ & RECIST \\
\hline $\begin{array}{l}\text { IPASS } \\
(2009)\end{array}$ & Mok & $\begin{array}{l}\text { Asian, nonsmokers or light smokers patients with stage IIIB or IV } \\
\text { adenocarcinoma of the lung who had no previous chemotherapy }\end{array}$ & First & $\begin{array}{l}\text { Gefitinib } 250 \mathrm{mg} / \text { day }(n=609) \\
\text { Carboplatin AUC }=5 \text { or } 6 \text { plus paclitaxel } 200 \mathrm{mg} / \mathrm{m}^{2} \\
\text { every } 3 \text { weeks }(n=608)\end{array}$ & RECIST \\
\hline
\end{tabular}

Abbreviations: NSCLC, non-small-cell lung cancer; EGFR, epidermal growth factor receptor; AUC, area under the curve; RECIST, response evaluation criteria in Solid tumors; WHO, world health organization.

(54 out of 237) in the chemotherapy arm. For erlotinib versus chemotherapy, RR was 2.41 (95\% CI, 1.68-3.47; $p<0.0001)$. ORR was $67.3 \%$ (187 patients of 278 for the erlotinib arm and $28.2 \%$ ( 75 out of 266 ) for the chemotherapy arm. Heterogeneity was high between studies $\left(I^{2}=78 \%\right)$.

In terms of progression free survival (PFS), the pooled hazard ratio (HR) for gefitinib as first-line HR was $0.62(95 \% \mathrm{CI}, 0.38-1.00$ (Figure 3$)$. In the afatinib analysis, HR was $0.40(95 \% \mathrm{CI}, 0.20-0.83)$. In the erlotinib one, HR was 0.28 (95\% CI, 0.17-0.45). Heterogeneity was high $\left(I^{2}=93 \%\right)$.

Accessing overall survival (OS), heterogeneity was very low $\left(I^{2}=0 \%\right)$. For Gefitinib HR was $0.91(95 \% \mathrm{CI}$, $0.82-1.02 ; p=0.11$ ) (Figure 4). For afatinib, HR was 1.01 $(95 \% \mathrm{CI}, 0.78-1.32 ; p=0.93)$ and 1.04 (95\% CI, $0.83-$ $1.31 ; p=0.72$ ) for erlotinib.

Most common adverse events of EGFR TKIs [26] were analysed (Figures 5-8). Diarrhoea of any grade was a common side effect for these patients. Comparing gefitinib with afatinib, RR was 0.51 (95\% CI, 0.47-
$0.54 ; p<0.00001)$; gefitinib with erlotinib, RR was 1.00 (95\% CI, 0.93-1.26; $p=0.03$ ); and afatinib with erlotinib, RR was 2.13 (95\% CI, $1.86-2.45 ; p<$ 0.00001).

The incidence of skin rash was also observed. In the indirect comparison, gefitinib versus afatinib showed RR of 0.82 (95\% CI, $0.77-0.87 ; p<0.00001)$. For gefitinib versus erlotinib, RR was 0.93 (95\% CI, 0.86-1.01; $p=$ $0.10)$. For afatinib versus erlotinib, RR was $1.14(95 \% \mathrm{CI}$, $1.05-1.23 ; p=0.001$ ) (Figure 6).

For the occurrence of stomatitis (Figure 7), the pooled RR for gefitinib versus afatinib was 0.33 (95\% CI, 0.29-0.38; $p<0.00001)$; gefitinib versus erlotinib, 2.31 (95\% CI, 1.44-3.70; $p=0.00015)$; afatinib versus erlotinib, 7.01 (95\% CI, 4.43-11.10; $p<0.00001)$.

Paronychia was also accessed (Figure 8). The indirect comparison showed RR of 0.34 (95\% CI, $0.28-0.41 ; p<0.00001)$ for gefitinib versus afatinib, 1.45 (95\% CI, $0.92-2.26 ; p=0.11$ ) for gefitinib versus erlotinib, and 4.29 (95\% CI, 2.80-6.57; $p<0.00001)$ for afatinib versus erlotinib. 
Table 2: Patient demographics and disease characteristics of included studies

\begin{tabular}{|c|c|c|c|c|c|c|c|}
\hline Characteristic & & $\begin{array}{l}\text { Gefitinib } \\
(n=968)\end{array}$ & $\begin{array}{l}\text { Control } \\
(n=958)\end{array}$ & $\begin{array}{l}\text { Erlotinib } \\
(n=278)\end{array}$ & $\begin{array}{l}\text { Control } \\
(n=266)\end{array}$ & $\begin{array}{l}\text { Afatinib } \\
(n=472)\end{array}$ & $\begin{array}{l}\text { Control } \\
(n=237)\end{array}$ \\
\hline \multirow[t]{2}{*}{ Sex } & Male & $213(22 \%)$ & $210(21.9 \%)$ & $104(37.4 \%)$ & $90(33.8 \%)$ & $170(36 \%)$ & $77(32.5 \%)$ \\
\hline & Female & $755(78 \%)$ & $748(78.1 \%)$ & $174(62.6 \%)$ & $176(66.2 \%)$ & $302(64 \%)$ & $160(67.5 \%)$ \\
\hline Age (median) $) \dagger$ & & 60.5 & 60 & 59.8 & 60 & 59.7 & 59.5 \\
\hline \multirow[t]{2}{*}{ Smoking status } & Never smoker & $866(89.5 \%)$ & $842(87.9 \%)$ & $195(70.1 \%)$ & $187(70.3 \%)$ & $336(71.2 \%)$ & $180(75.9 \%)$ \\
\hline & $\begin{array}{l}\text { Previous or } \\
\text { current smoker }\end{array}$ & $102(10.5 \%)$ & $116(12.1 \%)$ & $140(50.4 \%)$ & $79(29.7 \%)$ & $136(28.8 \%)$ & $57(24.1 \%)$ \\
\hline \multirow[t]{2}{*}{ ECOG } & $0-1$ & $892(92.1 \%)$ & $877(91.5 \%)$ & $252(90.6 \%)$ & $245(92.1 \%)$ & $472(100 \%)$ & $236(99.6 \%)$ \\
\hline & 2 & $76(7.9 \%)$ & $81(8.5 \%)$ & $26(9.4 \%)$ & $21(7.9 \%)$ & $0(0 \%)$ & $1(0.4 \%)$ \\
\hline \multirow[t]{3}{*}{ Histologic diagnosis } & Adenocarcinoma & $926(95.7 \%)$ & $934(97.5 \%)$ & $258(92.8 \%)$ & $241(90.6 \%)$ & $472(100 \%)$ & $237(100 \%)$ \\
\hline & Other & $39(4 \%)$ & $20(2.1 \%)$ & $20(7.2 \%)$ & $25(9.4 \%)$ & $0(0 \%)$ & $0(0 \%)$ \\
\hline & Unknown & $3(0.3 \%)$ & $3(0.3 \%)$ & $0(0 \%)$ & $0(0 \%)$ & $0(0 \%)$ & $0(0 \%)$ \\
\hline \multirow[t]{3}{*}{ EGFR mutation } & Positive & $358(37 \%)$ & $345(36 \%)$ & $278(100 \%)$ & $266(100 \%)$ & $472(100 \%)$ & $237(100 \%)$ \\
\hline & Negative & $118(12.2 \%)$ & $112(11.7 \%)$ & $0(0 \%)$ & $0(0 \%)$ & $0(0 \%)$ & $0(0 \%)$ \\
\hline & Unknown & $492(50.8 \%)$ & $501(52.3 \%)$ & $0(0 \%)$ & $0(0 \%)$ & $0(0 \%)$ & $0(0 \%)$ \\
\hline
\end{tabular}

Abbreviations: ECOG, Eastern Collaborative Oncology Group; EGFR, epidermal growth factor receptor.

EGFR TKI Control Risk Ratio Study or Subgroup Events Total Events Total Weight M-H, Random, 95\% Cl

Risk Ratio 6.1.1 Gefitinib vs. Chemotherapy

A

$\begin{array}{lrrrrrr}\text { First-SIGNAL (2012) } & 94 & 132 & 61 & 129 & 12.8 \% & 1.51[1.22,1.86] \\ \text { IPASS (2009) } & 262 & 609 & 196 & 608 & 13.6 \% & 1.33[1.15,1.55] \\ \text { NE.J002 (2010) } & 84 & 114 & 35 & 114 & 11.6 \% & 2.40[1.78,3.23] \\ \text { W.JTOG3405(2009) } & 36 & 58 & 19 & 59 & 9.7 \% & 1.93[1.26,2.94] \\ \text { Subtotal (95\% Cl) } & & 913 & & 910 & 47.7 \% & 1.69[1.31,2.19] \\ \text { Total events } & 476 & & 311 & & & \end{array}$

Total events

$476 \quad 311$

Heterogeneity: Tau $^{2}=0.05 ; \mathrm{Chi}^{2}=13.33, \mathrm{df}=3(\mathrm{P}=0.004) ; \mathrm{I}^{2}=77 \%$

Test for owerall effect: $Z=3.99$ ( $P<0.0001)$

B 6.1.2 Erlotinib vs. Chemotherapy

$\begin{array}{lrrrrrr}\text { ENSURE (2015) } & 69 & 110 & 36 & 107 & 11.5 \% & 1.86[1.38,2.52] \\ \text { EURTAC (2012) } & 50 & 86 & 13 & 87 & 8.1 \% & 3.89[2.28,6.63] \\ \text { OPTIMAL (2011) } & 68 & 82 & 26 & 72 & 11.2 \% & 2.30[1.66,3.17] \\ \text { Subtotal (95\% Cl) } & & 278 & & 266 & 30.7 \% & 2.41[1.68,3.47] \\ \text { Total ewents } & 187 & & 75 & & & \end{array}$

Heterogeneity: Tau $^{2}=0.07 ; \mathrm{Chi}^{2}=5.74, \mathrm{df}=2(\mathrm{P}=0.06) ; \mathrm{I}^{2}=65 \%$

Test for owerall effect: $Z=4.75$ ( $P=0.00001)$

C 6.1.3 Afatinib vs. Systemic treatment

$\begin{array}{lllllll}\text { LUX-Lung 3 (2013) } & 129 & 230 & 26 & 115 & 10.6 \% & 2.48[1.74,3.54] \\ \text { LUX-Lung 6 (2014) } & 162 & 242 & 28 & 122 & 11.0 \% & 2.92[2.08,4.09] \\ \text { Subtotal (95\% Cl) } & & 472 & & 237 & 21.6 \% & 2.70[2.12,3.45] \\ \text { Total events } & 291 & & 54 & & & \end{array}$

Heterogeneity: $\mathrm{Tau}^{2}=0.00 ; \mathrm{Chi}^{2}=0.42, \mathrm{df}=1(\mathrm{P}=0.52) ; \mathrm{i}^{2}=0 \%$

D Test for owerall effect: $Z=7.95$ ( $\mathrm{P}=0.00001$ )

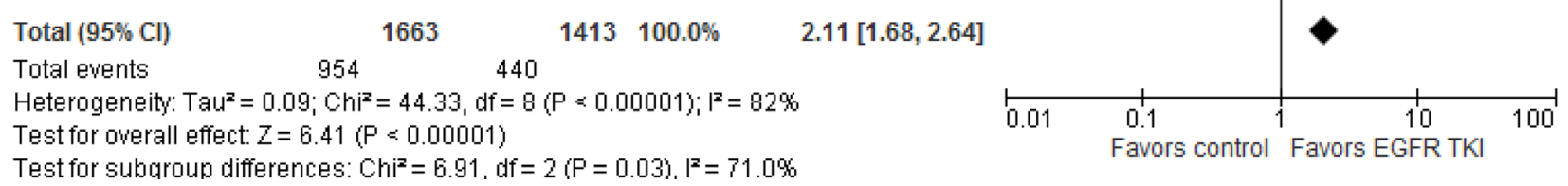

Figure 2: (A-D) Individual study and meta-estimate risk ratio of objective response ratio for gefitinib, afatinib, and erlotinib. ORR, overall response rate; PFS, progression-free-survival; OS, overall survival. 


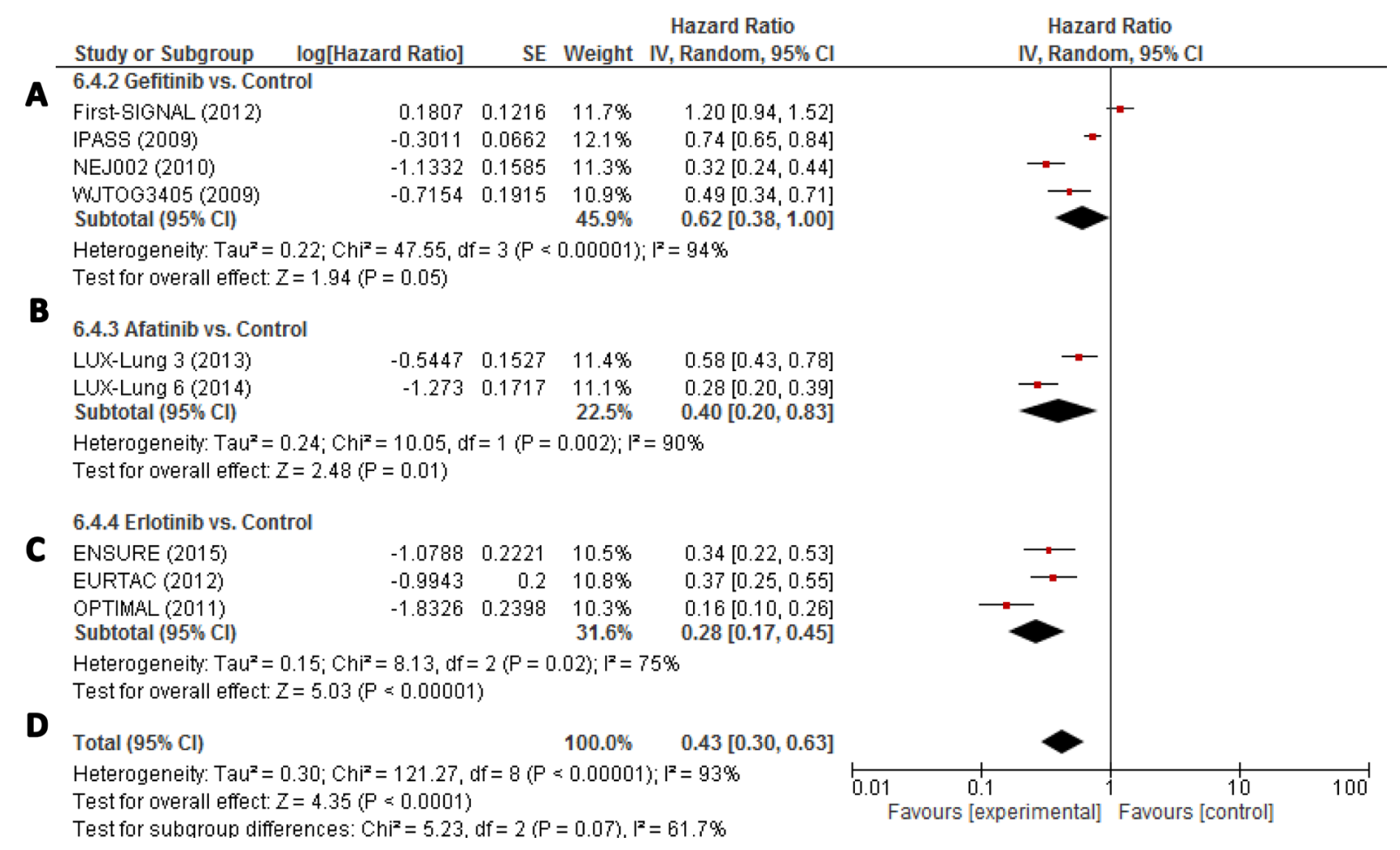

Figure 3: (A-D) Individual study hazard ratios with pooled estimation for progression-free survival for gefitinib, erlotinib, and afatinib. ORR, overall response rate; PFS, progression-free-survival; OS, overall survival.

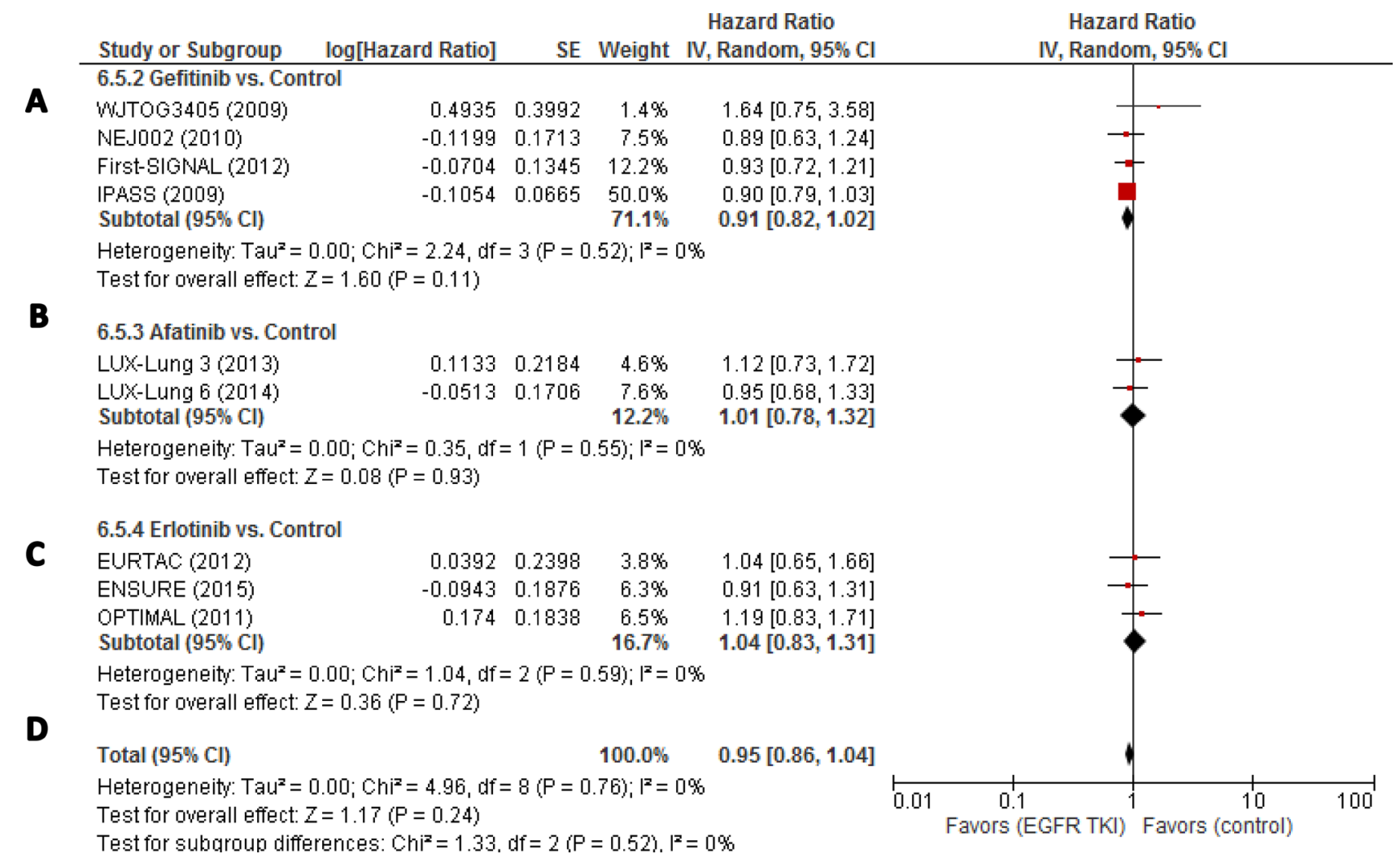

Figure 4: (A-D) Individual study hazard ratios with pooled estimation for overall survival for gefitinib, erlotinib, and afatinib. ORR, overall response rate; PFS, progression-free-survival; OS, overall survival. 


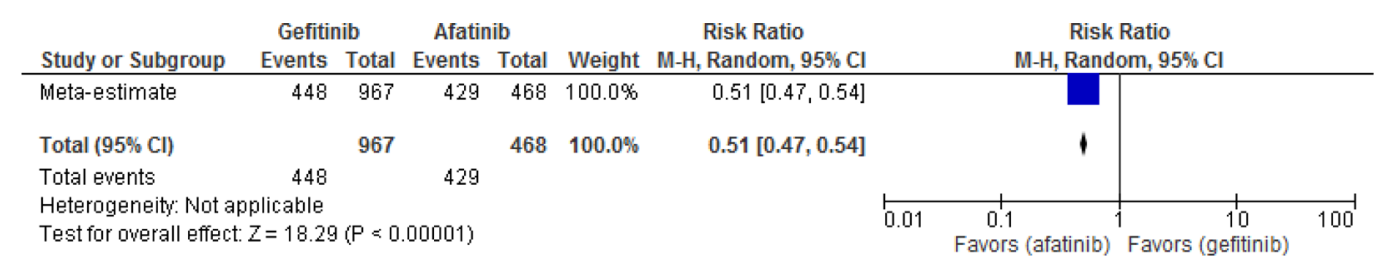

A Gefitinib versus afatinib.

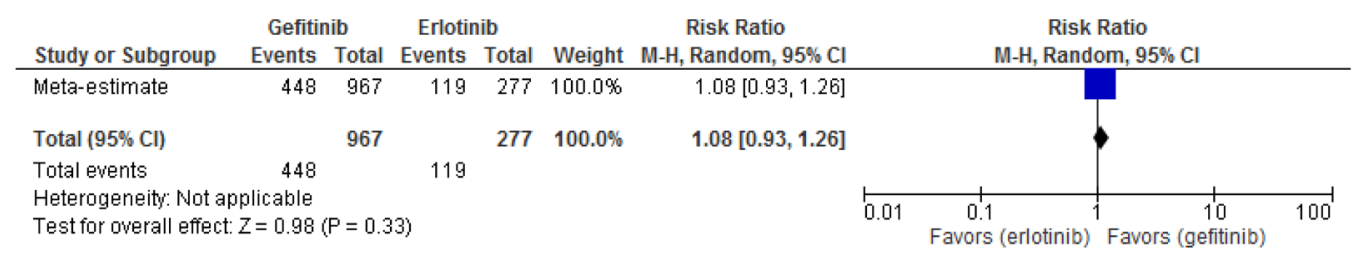

B Gefitinib versus erlotinib.

\begin{tabular}{|c|c|c|c|c|c|c|c|c|c|c|}
\hline \multirow[b]{2}{*}{ Study or Subgroup } & \multicolumn{2}{|c|}{ Afatinib } & \multicolumn{2}{|c|}{ Erlotinib } & \multirow[b]{2}{*}{ Weight } & \multirow{2}{*}{$\begin{array}{c}\text { Risk Ratio } \\
\text { M-H, Random, } 95 \% \mathrm{Cl}\end{array}$} & \multirow{2}{*}{\multicolumn{4}{|c|}{$\begin{array}{c}\text { Risk Ratio } \\
\text { M-H, Random, } 95 \% \mathrm{Cl}\end{array}$}} \\
\hline & Events & Total & Events & Total & & & & & & \\
\hline Meta-estimate & 429 & 468 & 119 & 277 & $100.0 \%$ & $2.13[1.86,2.45]$ & & & & \\
\hline Total $(95 \% \mathrm{Cl})$ & & 468 & & 277 & $100.0 \%$ & $2.13[1.86,2.45]$ & & & $\uparrow$ & \\
\hline Total events & 429 & & 119 & & & & & & & \\
\hline $\begin{array}{l}\text { Heterogeneity: Not a } \\
\text { Test for owerall effect }\end{array}$ & $\begin{array}{l}\text { plicable } \\
Z=10.73\end{array}$ & $3(P=0$ & $.00001)$ & & & & 0.01 & $\begin{array}{c}1 \\
0.1 \\
\text { Favors (erlotinib) }\end{array}$ & $10 \frac{10}{\text { Favors (afatinib) }}$ & 100 \\
\hline
\end{tabular}

\section{Afatinib versus erlotinib.}

Figure 5: (A-C) Pooled risk ratio of gefitinib, erlotinib, and afatinib indirectly compared for the ocurrence of diahrrea. EGFR, epidermal growth factor receptor; ORR, overall response rate; PFS, progression-free-survival; OS, overall survival.

\begin{tabular}{|c|c|c|c|c|c|c|c|c|c|c|}
\hline Study or Subgroup & \multicolumn{2}{|c|}{ Gefitinib } & \multicolumn{2}{|c|}{ Afatinib } & Weight & $\begin{array}{c}\text { Risk Ratio } \\
\text { M-H, Random, } 95 \% \mathrm{Cl}\end{array}$ & \multicolumn{4}{|c|}{$\begin{array}{c}\text { Risk Ratio } \\
\text { M-H, Random, } 95 \% \mathrm{Cl}\end{array}$} \\
\hline Meta-estimate & 672 & 967 & 397 & 468 & $100.0 \%$ & $0.82[0.77,0.87]$ & & & & \\
\hline Total $(95 \% \mathrm{Cl})$ & & 967 & & 468 & $100.0 \%$ & $0.82[0.77,0.87]$ & & 1 & & \\
\hline Total ewents & 672 & & 397 & & & & & & & \\
\hline $\begin{array}{l}\text { Heterogeneity: Not a } \\
\text { Test for overall effect }\end{array}$ & $\begin{array}{l}\text { plicable } \\
Z=6.90\end{array}$ & & 10001) & & & & 0.01 & $\begin{array}{c}0.1 \\
\text { Favors (afatinib) }\end{array}$ & \begin{tabular}{|cc}
1 \\
10 \\
Favors (gefitinib)
\end{tabular} & $\overrightarrow{100}$ \\
\hline
\end{tabular}

\section{A Gefitinib versus afatinib.}

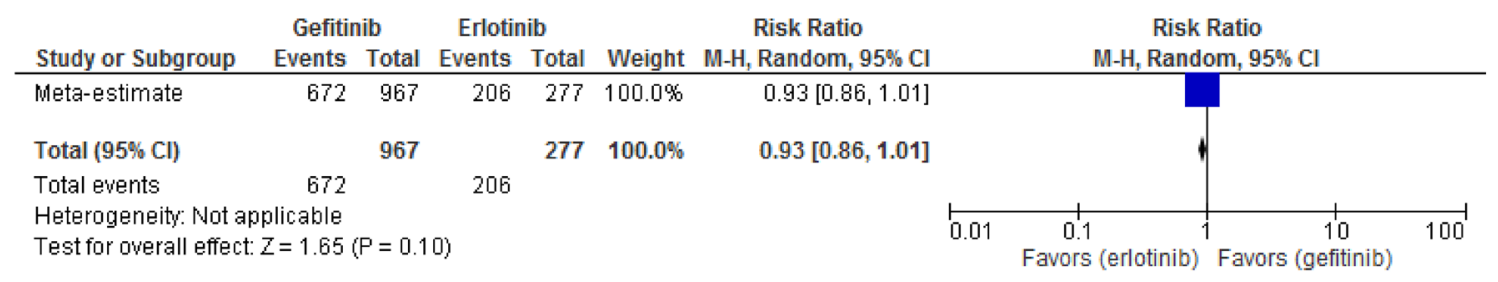

B Gefitinib versus erlotinib.

\begin{tabular}{|c|c|c|c|c|c|c|c|c|c|c|}
\hline \multirow[b]{2}{*}{ Study or Subgroup } & \multicolumn{2}{|c|}{ Afatinib } & \multicolumn{2}{|c|}{ Erlotinib } & \multirow[b]{2}{*}{ Weight } & \multirow{2}{*}{$\begin{array}{c}\text { Risk Ratio } \\
\text { M-H, Random, } 95 \% \mathrm{Cl}\end{array}$} & \multirow{2}{*}{\multicolumn{4}{|c|}{$\begin{array}{c}\text { Risk Ratio } \\
\text { M-H, Random, } 95 \% \mathrm{Cl}\end{array}$}} \\
\hline & Events & Total & Events & Total & & & & & & \\
\hline Weta-estimate & 397 & 468 & 206 & 277 & $100.0 \%$ & $1.14[1.05,1.23]$ & & & & \\
\hline Total $(95 \% \mathrm{Cl})$ & & 468 & & 277 & $100.0 \%$ & $1.14[1.05,1.23]$ & & & 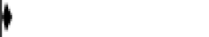 & \\
\hline Total events & 397 & & 206 & & & & & & & \\
\hline $\begin{array}{l}\text { Heterogeneity: Not a } \\
\text { Test for overall effect }\end{array}$ & $\begin{array}{l}\text { olicable } \\
z=3.260\end{array}$ & $P=0.0$ & & & & & 0.01 & 0.1 ors (erlotinib) & Favors (afatinib) & 100 \\
\hline
\end{tabular}

\section{Afatinib versus erlotinib.}

Figure 6: (A-C) Pooled risk ratio of gefitinib, erlotinib, and afatinib indirectly compared for the ocurrence of skin rash. 


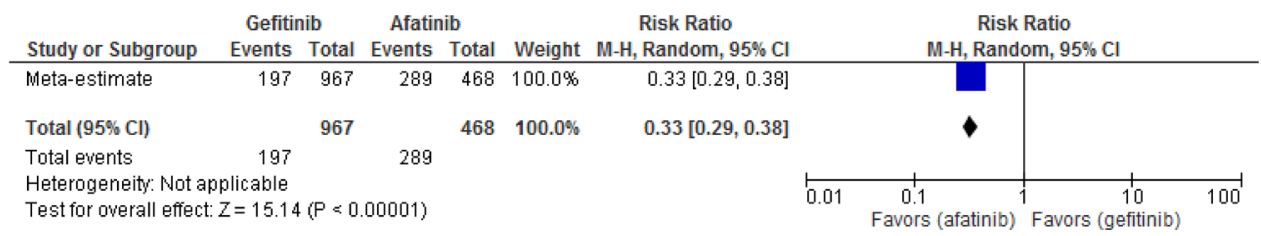

A Gefitinib versus afatinib.

\begin{tabular}{|c|c|c|c|c|c|c|c|c|c|c|}
\hline \multirow[b]{2}{*}{ Study or Subgroup } & \multicolumn{2}{|c|}{ Gefitinib } & \multicolumn{2}{|c|}{ Erlotinib } & \multicolumn{2}{|r|}{ Risk Ratio } & \multirow{2}{*}{\multicolumn{4}{|c|}{$\begin{array}{c}\text { Risk Ratio } \\
\text { M-H, Random, } 95 \% \mathrm{Cl}\end{array}$}} \\
\hline & Events & Total & Events & Total & Weight & M-H, Random, $95 \% \mathrm{Cl}$ & & & & \\
\hline Meta-estimate & 197 & 967 & 17 & 193 & $100.0 \%$ & $2.31[1.44,3.70]$ & & & & \\
\hline Total $(95 \% \mathrm{Cl})$ & & 967 & & 193 & $100.0 \%$ & $2.31[1.44,3.70]$ & & & & \\
\hline Total events & 197 & & 17 & & & & & & & \\
\hline $\begin{array}{l}\text { Heterogeneity: Not a } \\
\text { Test for overall effect }\end{array}$ & $\begin{array}{l}\text { plicable } \\
z=3.49\end{array}$ & $P=0.0$ & 1005) & & & & 0.01 & $\begin{array}{c}0.1 \\
\text { Favors (erlotinib) }\end{array}$ & 10 & 100 \\
\hline
\end{tabular}

B Gefitinib versus erlotinib.

\begin{tabular}{|c|c|c|c|c|c|c|c|c|c|c|}
\hline Study or Subgroup & \multicolumn{2}{|c|}{ Afatinib } & \multicolumn{2}{|c|}{ Erlotinib } & Weight & $\begin{array}{c}\text { Risk Ratio } \\
\mathrm{M}-\mathrm{H}, \text { Random, } 95 \% \mathrm{Cl}\end{array}$ & \multicolumn{3}{|c|}{$\begin{array}{c}\text { Risk Ratio } \\
\text { M-H. Random, } 95 \% \mathrm{Cl}\end{array}$} & \\
\hline Meta-estimate & 289 & 468 & 17 & 193 & $100.0 \%$ & $7.01[4.43,11.10]$ & & & & \\
\hline Total $(95 \% \mathrm{Cl})$ & & 468 & & 193 & $100.0 \%$ & $7.01[4.43,11.10]$ & & & & \\
\hline Total events & 289 & & 17 & & & & & & & \\
\hline $\begin{array}{l}\text { Heterogeneity: Not a } \\
\text { Test for owerall effec }\end{array}$ & $\begin{array}{l}\text { plicable } \\
Z=8.31\end{array}$ & $P=0.0$ & (0001) & & & & 0.01 & $\begin{array}{c}1 \\
0.1 \\
\text { Favors (erlotinib) }\end{array}$ & Favors (a) & 100 \\
\hline
\end{tabular}

C Afatinib versus erlotinib.

Figure 7: (A-C) Pooled risk ratio of gefitinib, erlotinib, and afatinib indirectly compared for the ocurrence of stomatitis.

\begin{tabular}{|c|c|c|c|c|c|c|c|c|c|c|}
\hline Study or Subgroup & \multicolumn{2}{|c|}{ Gefitinib } & \multicolumn{2}{|c|}{ Afatinib } & Weight & $\begin{array}{c}\text { Risk Ratio } \\
\text { M-H, Random, } 95 \% \mathrm{Cl}\end{array}$ & \multicolumn{4}{|c|}{$\begin{array}{c}\text { Risk Ratio } \\
\text { M-H, Random, } 95 \% \mathrm{Cl}\end{array}$} \\
\hline Weta-estimate & 121 & 808 & 208 & 468 & $100.0 \%$ & $0.34[0.28,0.41]$ & & & & \\
\hline Total $(95 \% \mathrm{Cl})$ & & 808 & & 468 & $100.0 \%$ & $0.34[0.28,0.41]$ & & & & \\
\hline Total events & 121 & & 208 & & & & & & & \\
\hline $\begin{array}{l}\text { Heterogeneity: Not a } \\
\text { Test for overall effect }\end{array}$ & $\begin{array}{l}\text { plicable } \\
Z=11.05\end{array}$ & $(\mathrm{P}<0$ & $.00001)$ & & & & $\stackrel{\longmapsto}{\circ .01}$ & $\begin{array}{c}0.1 \\
\text { Favors (afatinib) }\end{array}$ & $\frac{10}{10}$ & $\overrightarrow{100}$ \\
\hline
\end{tabular}

\section{A Gefitinib versus afatinib.}

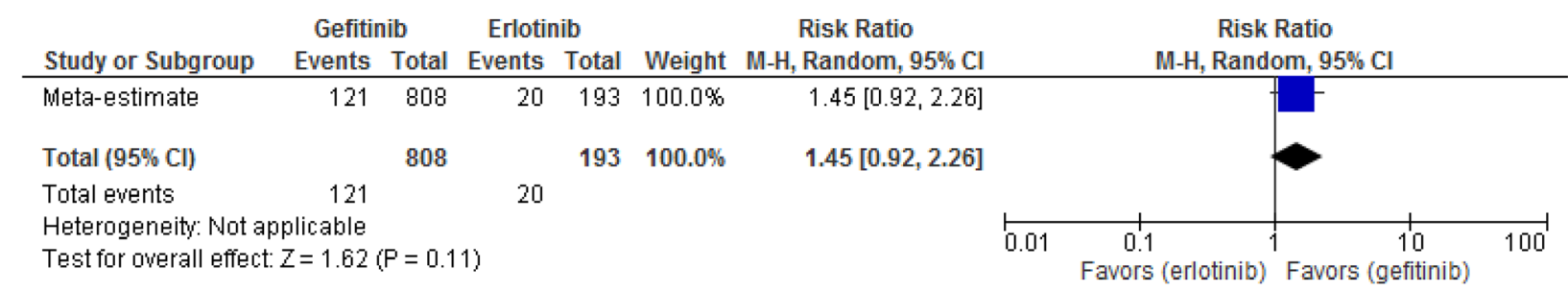

B Gefitinib versus erlotinib.

\begin{tabular}{|c|c|c|c|c|c|c|c|c|c|c|}
\hline Study or Subgroup & \multicolumn{2}{|c|}{ Afatinib } & \multicolumn{2}{|c|}{ Erlotinib } & Weight & $\begin{array}{c}\text { Risk Ratio } \\
\text { M-H, Random, } 95 \% \mathrm{Cl}\end{array}$ & \multicolumn{4}{|c|}{$\begin{array}{c}\text { Risk Ratio } \\
\text { M-H, Random, } 95 \% \mathrm{Cl}\end{array}$} \\
\hline Meta-estimate & 208 & 469 & 20 & 193 & $100.0 \%$ & $4.29[2.80,6.57]$ & & & & \\
\hline Total $(95 \% \mathrm{Cl})$ & & 468 & & 193 & $100.0 \%$ & $4.29[2.80,6.57]$ & & & & \\
\hline Total events & 208 & & 20 & & & & & & & \\
\hline $\begin{array}{l}\text { Heterogeneity: Not a } \\
\text { Test for overall effect }\end{array}$ & $\begin{array}{l}\text { olicable } \\
z=6.68\end{array}$ & $P<0.0$ & $0001)$ & & & & 0.0 & $\begin{array}{c}0.1 \\
\text { Favors (erlotinib) }\end{array}$ & Favors (afatinib) & 100 \\
\hline
\end{tabular}

\section{Afatinib versus erlotinib.}

Figure 8: (A-C) Pooled risk ratio of gefitinib, erlotinib, and afatinib indirectly compared for the ocurrence of paronychia. 


\section{DISCUSSION}

Currently, the landscape of NSCLC treatment is changing. Most recently, the use of EGFR TKI agents for patients harbouring activating mutations of EGFR (exons 18-21) is the standard of care. Several drugs have been approved in this setting, including gefitinib, erlotinib and recently afatinib. In this meta-analysis, gefitinib, erlotinib, and afatinib were superior in terms of objective response rate and progression free survival than platinum-based chemotherapy, but, as expected due to the cross-over effect, there was no statistically significant differences in terms of OS for either of the three drugs. Overall, gefitinib had the most consistent efficacy profile from a statistical point of view, and erlotinib had the best efficacy profile in terms of comparative improvement of PFS.

Our results challenge the recently reported results of LUX-Lung 7 [27], a phase $2 b$ trial comparing afatinib with gefitinib as first-line treatment in patients harboring EGFR mutations, that showed improvement in PFS and ORR with afatinib over gefitinib. Nevertheless, previous meta-analysis [28-30] evaluating first-line therapies of EGFR TKIs in EGFR mutation positive patients had not confirmed the results of this study. Although LUX-LUNG 7 is the only prospective, randomized clinical trial, it also harboured several drawbacks, including the small number of events, the lack of statistical power and the three co-primary endpoints. Our meta-analysis, on the other hand, is a retrospective collective analysis of data, but includes a large number of patients and possesses robust statistical power.

Afatinib was more likely to be related to adverse events, as expected because of its irreversible binding to ATP site of EGFR, HER2 and HER4, in contrast to the reversible nature of binding of gefitinib and erlotinib. [3132]. Differences between gefitinib and erlotinib were not statistically significant, except for paronychia, which was more frequent with erlotinib.

Limitations of our study include its retrospective nature and the indirect comparison between gefitinib, erlotinib and afatinib, since there is a paucity of head-to-head clinical trials, with the exception of LUX-LUNG 7; the high heterogeneity obtained during the data analysis; and the relative paucity of studies evaluating afatinib. Strengths of our study included the large number of patients, the robust statistical design and the broader range of therapies included, as we present data on the three approved first-line drugs.

Future studies are warranted to associate each type of EGFR-activating mutation to the efficacy of a specific treatment and to compare new drugs, as osimertinib, with first and second generation TKIs.

In conclusion, gefitinib, erlotinib, and afatinib are effective in the treatment of NSCLC in terms of progression free survival and objective response rate. Gefitinib had the most consistent efficacy profile from a statistical point of view, and erlotinib had the best efficacy profile in terms of comparative improvement of PFS. As Afatinib still remains the agent with best CSF penetration, we suggest its use is limited to patients presenting with brain metastasis. We suggest the use of Gefitinib in patients without CNS involvement. Faced with the impossibility to dose-reduce Gefitinib, Erlotinib represents a tolerable and effective alternative to Afatinib and Gefitinib if response to EGFR inhibition is considered still effective.

\section{ACKNOWLEDGMENTS}

Logistic provision from the Cearense School of Oncology, Ceará Cancer Institute, Haroldo Juaçaba Hospital, Fortaleza, Ceará, Brazil, and The Clatterbridge Cancer Centre NHS Foundation Trust, Wirral \& Liverpool, England, United Kingdom.

\section{CONFLICTS OF INTEREST}

RAM has received honoraria from Pfizer Advisory Board, Zodiac advisory board, AstraZeneca, Novartis, National Science Centre, Krakow, Poland, and educational grant from Pierre Fabre, Amgem. RAM is ad hoc consultant at Ministry of Health, Brasília, Brazil. The other authors have no conflicts of interest in this manuscript. GM has received honoraria from Bristol Myers Squibb, Roche and AstraZeneca.

\section{FUNDING}

This research did not receive any specific grant from funding agencies in the public, commercial, or not-forprofit sectors.

\section{REFERENCES}

1. Ferlay J, Soerjomataram I, Dikshit R, Eser S, Mathers C, Rebelo M, Parkin DM, Forman D, Bray F. Cancer incidence and mortality worldwide: sources, methods and major patterns in GLOBOCAN 2012. Int J Cancer. 2015; 5:E359-386.

2. Midha A, Dearden S, McCormack R. EGFR mutation incidence in non-small-cell lung cancer of adenocarcinoma histology: a systematic review and global map by ethnicity (mutMapII). Am J Cancer Res. 2015; 9:2892-2911.

3. Tsiambas E, Lefas AY, Georgiannos SN, Ragos V, Fotiades PP, Grapsa D, Stamatelopoulos A, Kavantzas N, Patsouris E, Syrigos K. EGFR gene deregulation mechanisms in lung adenocarcinoma: A molecular review. Pathol Res Pract. 2016; 8:672-677.

4. Wakeling AE, Guy SP, Woodburn JR, Ashton SE, Curry BJ, Barker AJ, Gibson KH. ZD1839 (Iressa): an orally active inhibitor of epidermal growth factor signaling with potential for cancer therapy. Cancer Res. 2002; 20:5749-5754.

5. Steuer CE, Khuri FR, Ramalingam SS. The next generation of epidermal growth factor receptor tyrosine kinase inhibitors in the treatment of lung cancer. Cancer. 2015; 8:E1-6. 
6. Chung C. Tyrosine kinase inhibitors for epidermal growth factor receptor gene mutation-positive non-small cell lung cancers: an update for recent advances in therapeutics. J Oncol Pharm Pract. 2016; 3:461-476.

7. Greig SL. Osimertinib: First Global Approval. Drugs. 2016; 2:263-273.

8. Tan WL, Jain A, Takano A, Newell EW, Iyer NG, Lim WT, Tan EH, Zhai W, Hillmer AM, Tam WL, Tan DSW. Novel therapeutic targets on the horizon for lung cancer. Lancet Oncol 2016, 8:e347-362.

9. Reck M, Popat S, Reinmuth N, De Ruysscher D, Kerr KM, Peters S, and ESMO Guidelines Working Group. Metastatic non-small-cell lung cancer (NSCLC): ESMO Clinical Practice Guidelines for diagnosis, treatment and follow-up. Ann Oncol. 2014; 25:iii27-39.

10. Zhang M, Guo H, Zhao S, Wang Y, Yang M, Yu J, Yan Y. Efficacy of epidermal growth factor receptor inhibitors in combination with chemotherapy in advanced non-small cell lung cancer: A meta-analysis of randomized controlled trials. Oncotarget. 2016; 7:39823-39833. http://doi. org/10.18632/oncotarget.9503.

11. Thatcher N, Chang A, Parikh P, Rodrigues Pereira J, Ciuleanu T, von Pawel J, Thongprasert S, Tan EH, Pemberton K, Archer V, Carroll K. Gefitinib plus best supportive care in previously treated patients with refractory advanced non-small-cell lung cancer: results from a randomised, placebo-controlled, multicentre study (Iressa Survival Evaluation in Lung Cancer). Lancet. 2005; 9496:1527-1537.

12. Zhou C, Wu YL, Chen G, Feng J, Liu XQ, Wang C, Zhang S, Wang J, Zhou S, Ren S, Lu S, Zhang L, Hu C, et al. Erlotinib versus chemotherapy as first-line treatment for patients with advanced EGFR mutation-positive non-smallcell lung cancer (OPTIMAL, CTONG-0802): a multicentre, open-label, randomised, phase 3 study. Lancet Oncol. 2011; 8:735-742.

13. Kim ES, Hirsh V, Mok T, Socinski MA, Gervais R, Wu YL, Li LY, Watkins CL, Sellers MV, Lowe ES, Sun Y, Liao ML, Osterlind K, et al. Gefitinib versus docetaxel in previously treated non-small-cell lung cancer (INTEREST): a randomised phase III trial. Lancet. 2008; 9652:1809-1818.

14. Mok TS, Wu YL, Thongprasert S, Yang CH, Chu DT, Saijo N, Sunpaweravong P, Han B, Margono B, Ichinose Y, Nishiwaki Y, Ohe Y, Yang JJ, et al. Gefitinib or carboplatinpaclitaxel in pulmonary adenocarcinoma. N Engl J Med. 2009; 10:947-957.

15. Maemondo M, Inoue A, Kobayashi K, Sugawara S, Oizumi $\mathrm{S}$, Isobe $\mathrm{H}$, Gemma $\mathrm{A}$, Harada $\mathrm{M}$, Yoshizawa $\mathrm{H}$, Kinoshita I, Fujita Y, Okinaga S, Hirano H, et al. Gefitinib or chemotherapy for non-small-cell lung cancer with mutated EGFR. N Engl J Med. 2010; 25:2380-2388.

16. Mitsudomi T, Morita S, Yatabe Y, Negoro S, Okamoto I, Tsurutani J, Seto T, Satouchi M, Tada H, Hirashima T, Asami K, Katakami N, Takada M, et al. Gefitinib versus cisplatin plus docetaxel in patients with non-small-cell lung cancer harbouring mutations of the epidermal growth factor receptor (WJTOG3405): an open label, randomised phase 3 trial. Lancet Oncol. 2010; 2:121-128.

17. Han JY, Park K, Kim SW, Lee DH, Kim HY, Kim HT, Ahn MJ, Yun T, Ahn JS, Suh C, Lee JS, Yoon SJ, Han JH, et al. First-SIGNAL: first-line single-agent iressa versus gemcitabine and cisplatin trial in never-smokers with adenocarcinoma of the lung. J Clin Oncol. 2012; 10:11221128.

18. Miller VA, Hirsh V, Cadranel J, Chen YM, Park K, Kim SW, Zhou C, Su WC, Wang M, Sun Y, Heo DS, Crino L, Tan EH, et al. Afatinib versus placebo for patients with advanced, metastatic non-small-cell lung cancer after failure of erlotinib, gefitinib, or both, and one or two lines of chemotherapy (LUX-Lung 1): a phase 2b/3 randomised trial. Lancet Oncol. 2012; 5:528-538.

19. Wu YL, Zhou C, Hu CP, Feng J, Lu S, Huang Y, Li W, Hou M, Shi JH, Lee KY, Xu CR, Massey D, Kim M, et al. Afatinib versus cisplatin plus gemcitabine for first-line treatment of Asian patients with advanced non-small-cell lung cancer harbouring EGFR mutations (LUX-Lung 6): an open-label, randomised phase 3 trial. Lancet Oncol. 2014; 2:213-222.

20. Rosell R, Carcereny E, Gervais R, Vergnenegre A, Massuti B, Felip E, Palmero R, Garcia-Gomez R, Pallares C, Sanchez JM, Porta R, Cobo M, Garrido P, et al. Erlotinib versus standard chemotherapy as first-line treatment for European patients with advanced EGFR mutation-positive non-smallcell lung cancer (EURTAC): a multicentre, open-label, randomised phase 3 trial. Lancet Oncol. 2012; 3:239-246.

21. Therasse P, Arbuck SG, Eisenhauer EA, Wanders J, Kaplan RS, Rubinstein L, Verweij J, Van Glabbeke M, van Oosterom AT, Christian MC, Gwyther SG. New guidelines to evaluate the response to treatment in solid tumors. European Organization for Research and Treatment of Cancer, National Cancer Institute of the United States, National Cancer Institute of Canada. J Natl Cancer Inst. 2000; 3:205-216.

22. Higgins JP, Green S. Cochrane Handbook for Systematic Reviews of Interventions: Cochrane Book Series. Chichester, UK. 2008.

23. Kuritz SJ, Landis JR, Koch GG. A general overview of Mantel-Haenszel methods: applications and recent developments. Annu Rev Public Health. 1988; 9:123-60.

24. Sequist LV, Yang JC, Yamamoto N, O'Byrne K, Hirsh V, Mok T, Geater SL, Orlov S, Tsai CM, Boyer M, Su WC, Bennouna J, Kato T, et al. Phase III study of afatinib or cisplatin plus pemetrexed in patients with metastatic lung adenocarcinoma with EGFR mutations. J Clin Oncol. 2013; 27:3327-3334.

25. Wu YL, Zhou C, Liam CK, Wu G, Liu X, Zhong Z, Lu S, Cheng Y, Han B, Chen L, Huang C, Qin S, Zhu Y, et al. First-line erlotinib versus gemcitabine/cisplatin in patients with advanced EGFR mutation-positive non-small-cell lung cancer: analyses from the phase III, randomized, open-label, ENSURE study. Ann Oncol. 2015; 9:1883-1889. 
26. Melosky B, Hirsh V. Management of Common Toxicities in Metastatic NSCLC Related to Anti-Lung Cancer Therapies with EGFR-TKIs. Front Oncol. 2014; 4:238.

27. Park K, Tan EH, O'Byrne K, Zhang L, Boyer M, Mok T, Hirsh V, Yang JC, Lee KH, Lu S, Shi Y, Kim SW, Laskin $\mathrm{J}$, et al. Afatinib versus gefitinib as first-line treatment of patients with EGFR mutation-positive non-small-cell lung cancer (LUX-Lung 7): a phase 2B, open-label, randomised controlled trial. Lancet Oncol. 2016; 5:577-589.

28. Haaland B, Tan PS, de Castro G, Lopes G. Meta-analysis of first-line therapies in advanced non-mall-cell lung cancer harboring EGFR-activating mutations. J Thorac Oncol. 2014; 6:805-811.

29. Haspinger ER, Agustoni F, Torri V, Gelsomino F, Platania M, Zilembo N, Gallucci R, Garassino MC, Cinquini M. Is there evidence for different effects among EGFR-TKIs?
Systematic review and meta-analysis of EGFR tyrosine kinase inhibitors (TKIs) versus chemotherapy as first-line treatment for patients harboring EGFR mutations. Crit Rev Oncol Hematol. 2015; 2:213-227.

30. Greenhalgh J, Dwan K, Boland A, Bates V, Vecchio F, Dundar Y, Jain P, Green JA. First-line treatment of advanced epidermal growth factor receptor (EGFR) mutation positive non-squamous non-small cell lung cancer. Cochrane Database Syst Rev. 2016; 5:CD010383.

31. Wind S, Schnell D, Ebner T, Freiwald M, Stopfer P. Clinical Pharmacokinetics and Pharmacodynamics of Afatinib. Clin Pharmacokinet. 2016.

32. Takeda M, Okamoto I, Nakagawa K. Pooled safety analysis of EGFR-TKI treatment for EGFR mutation-positive nonsmall cell lung cancer. Lung Cancer. 2015; 1:74-79. 